\title{
Prevalence and Characteristics of Subjective Cognitive Decline Among Unpaid Caregivers Aged $\geq 45$ Years — 22 States, 2015-2019
}

\author{
Eva M. Jeffers, $\mathrm{MPH}^{1,2}$; Erin D. Bouldin, PhD ${ }^{1,3}$; Lisa C. McGuire, $\mathrm{PhD}^{1}$; Kenneth A. Knapp, PhD ${ }^{4}$; Roshni Patel, MPH ; Dana Guglielmo, MPH ${ }^{1,2}$; \\ Christopher A. Taylor, $\mathrm{PhD}^{1}$; Janet B. Croft, $\mathrm{PhD}^{1}$
}

Approximately $20 \%$ of U.S. adults are unpaid caregivers (caregivers) (1) who provide support to a family member or friend with a health condition or disability. Although there are benefits to caregiving, it can negatively affect caregivers' physical and mental health (2-4). Much of the assistance caregivers provide, such as administering medications or financial management, relies on cognitive ability, but little is known about caregivers' cognitive functioning. Subjective cognitive decline (SCD), the self-reported experience of worsening or more frequent confusion or memory loss over the past year (5), could affect caregivers' risk for adverse health outcomes and affect the quality of care they provide. CDC analyzed SCD among caregivers aged $\geq 45$ years through a cross-sectional analysis of data from 22 states in the 2015-2019 Behavioral Risk Factor Surveillance System (BRFSS). Among adults aged $\geq 45$ years, SCD was reported by $12.6 \%$ of caregivers who provided care to a family member or friend with a health condition or disability in the past 30 days compared with $10.2 \%$ of noncaregivers $(\mathrm{p}<0.001)$. Caregivers with SCD were more likely to be employed, men, aged 45-64 years, and have chronic health conditions than were noncaregivers with SCD. Caregivers with SCD were more likely to report frequent mental distress, a history of depression, and frequent activity limitations than were caregivers without SCD. SCD among caregivers could adversely affect the quality of care provided to care recipients. Understanding caregivers' cognitive health and the types of care provided is critical to maintaining the health, well-being, and independence of the caregiving dyad. Health care professionals can support patients and their patients' caregivers by increasing awareness among caregivers of the need to monitor their own health. The health care team can work with caregivers to identify potential treatments and access supports that might help them in their caregiving role and compensate for SCD.*

BRFSS is a cross-sectional, random-digit-dialed, annual telephone survey of noninstitutionalized U.S. adults aged $\geq 18$ years. BRFSS is conducted by state and territorial health departments, and data are weighted to make estimates representative of each state. Combined (landline and mobile) median

$\overline{{ }^{*} \text { https://www.cdc.gov/aging/publications/features/caring-for-yourself.html }}$

\section{INSIDE}

1597 Health Care Access and Use Among Adults with Diabetes During the COVID-19 Pandemic United States, February-March 2021

1603 Automated Digital Notification of COVID-19 Diagnoses Through Text and Email Messaging North Carolina, December 2020-January 2021

1608 Incidence of SARS-CoV-2 Infection, Emergency Department Visits, and Hospitalizations Because of COVID-19 Among Persons Aged $\geq 12$ Years, by COVID-19Vaccination Status - Oregon and Washington, July 4-September 25, 2021

1613 Impact of Hospital Strain on Excess Deaths During the COVID-19 Pandemic — United States, July 2020-July 2021

1617 Notes from the Field: Acute Nonviral Hepatitis Linked to a Brand of Alkaline Bottled WaterClark County, Nevada and California, 2020

1621 QuickStats

Continuing Education examination available at https://www.cdc.gov/mmwr/mmwr_continuingEducation.html

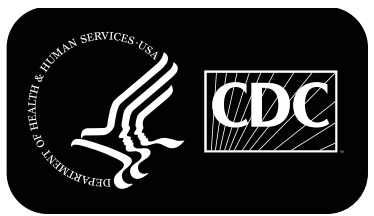

U.S. Department of Health and Human Services Centers for Disease Control and Prevention 
response rates for 2015-2019 ranged from $45.9 \%$ (2017) to 49.9\% (2018). ${ }^{\dagger}$ Among 22 states ${ }^{\S}$ in which BRFSS respondents were asked both the caregiving and cognitive decline questions in the same survey year during 2015-2019, the most recent year's data were analyzed for this study.

Respondents were classified as caregivers if they responded affirmatively when asked whether they had provided care to a family member or friend with a health condition or disability in the past 30 days. These respondents were then asked seven more questions about the care recipient and the type and duration of care provided (1). Personal care tasks included administering medications, feeding, dressing, and bathing; household tasks included cleaning, managing money, and preparing meals. Respondents were classified as experiencing SCD if they responded affirmatively when asked if they had experienced worsening or more frequent confusion or memory loss in the past 12 months.

\footnotetext{
$\dagger^{\dagger}$ https://www.cdc.gov/brfss/annual_data/2019/pdf/2019-response-rates-table-508.pdf; https://www.cdc.gov/brfss/annual_data/2018/pdf/2018-response-rates-table-508.pdf; https://www.cdc.gov/brfss/annual_data/2017/pdf/2017-response-rates-table-508.pdf; https://www.cdc.gov/brfss/annual_data/2016/pdf/2016_ResponseRates_Table.pdf; https://www.cdc.gov/brfss/annual_data/2015/2015_ResponseRates.html

$\$$ The following 22 U.S. states that included both caregiving and SCD modules in the same survey year during 2015-2019 are included (most recent year used): Alabama (2015), Florida (2015), Hawaii (2017), Illinois (2015), Iowa (2015), Louisiana (2015), Maryland (2019), Mississippi (2015), Missouri (2016), Montana (2016), Nebraska (2015), New Jersey (2018), New York (2019), Oregon (2019), South Carolina (2015), Tennessee (2019), Texas (2019), Utah (2019), Virginia (2019), West Virginia (2015), Wisconsin (2015), and Wyoming (2015).

https:/www.cdc.gov/aging/publications/BRFSS-caregiver-brief-508.pdf
}

Weighted, unadjusted prevalence of SCD by caregiver status was estimated among 93,604 community-dwelling respondents aged $\geq 45$ years and among a subgroup of 21,238 $(23.0 \%)$ caregivers, by sociodemographic, health-related, and caregiving-related characteristics. The distribution of these characteristics was estimated among caregivers by SCD status. Complex survey data methods were used to estimate weighted percentages and corresponding 95\% CIs using SAS-callable SUDAAN survey procedures (version 9.4; SAS Institute). T-tests were used to determine statistically significant differences between caregivers and noncaregivers with SCD, and modified Rao-Scott chi-square tests were used to estimate statistical differences between proportions of caregivers with and without SCD for each selected characteristic. P-values $<0.05$ were considered statistically significant for both tests. The relative standard error for all estimates was $<30 \%$. This activity was reviewed by CDC and was conducted consistent with applicable federal law and CDC policy.**

During 2015-2019, 23.0\% (95\% CI $=22.5 \%-23.6 \%)$ of U.S. adults (approximately 13 million) aged $\geq 45$ years in 22 states were caregivers. Among caregivers, the overall prevalence of SCD was $12.6 \%$ and varied by state, ranging from $9.8 \%$ (New Jersey) to $17.3 \%$ (Louisiana) (Table 1). In comparison, the prevalence of SCD among noncaregivers was $10.2 \%(\mathrm{p}<0.001)$ (Table 2$)$. Prevalence of SCD did not differ

** 45 C.F.R. part 46.102(l)(2), 21 C.F.R. part 56; 42 U.S.C. Sect. 241(d); 5 U.S.C. Sect. 552a; 44 U.S.C. Sect. 3501 et seq.

The MMWR series of publications is published by the Center for Surveillance, Epidemiology, and Laboratory Services, Centers for Disease Control and Prevention (CDC), U.S. Department of Health and Human Services, Atlanta, GA 30329-4027.

Suggested citation: [Author names; first three, then et al., if more than six.] [Report title]. MMWR Morb Mortal Wkly Rep 2021;70:[inclusive page numbers]

$$
\begin{gathered}
\text { Centers for Disease Control and Prevention } \\
\text { Rochelle P. Walensky, MD, MPH, Director } \\
\text { Debra Houry, MD, MPH, Acting Principal Deputy Director } \\
\text { Rebecca Bunnell, PhD, MEd, Director, Office of Science and Surveillance } \\
\text { Jennifer Layden, MD, PhD, Deputy Director, Office of Science } \\
\text { Michael F. Iademarco, MD, MPH, Director, Center for Surveillance, Epidemiology, and Laboratory Services }
\end{gathered}
$$

MMWR Editorial and Production Staff (Weekly)

Charlotte K. Kent, PhD, MPH, Editor in Chief Jacqueline Gindler, MD, Editor

Brian A. King, PhD, MPH, Guest Science Editor

Paul Z. Siegel, MD, MPH, Associate Editor

Mary Dott, MD, MPH, Online Editor

Terisa F. Rutledge, Managing Editor

Teresa M. Hood, MS, Lead Technical Writer-Editor

Leigh Berdon, Glenn Damon, Soumya Dunworth, PhD,

Tiana Garrett-Cherry, PhD, MPH, Srila Sen, MA

Stacy Simon, MA, Morgan Thompson, Technical Writer-Editors

Matthew L. Boulton, MD, MPH

Carolyn Brooks, ScD, MA

Jay C. Butler, MD

Virginia A. Caine, MD

Jonathan E. Fielding, MD, MPH, MBA

David W. Fleming, MD
Martha F. Boyd, Lead Visual Information Specialist

Alexander J. Gottardy, Maureen A. Leahy,

Julia C. Martinroe, Stephen R. Spriggs, Tong Yang, Visual Information Specialists

Quang M. Doan, MBA, Phyllis H. King,

Terraye M. Starr, Moua Yang,

Information Technology Specialists
Ian Branam, MA,

Acting Lead Health Communication Specialist Shelton Bartley, MPH, Leslie Hamlin,

Lowery Johnson, Amanda Ray,

Health Communication Specialists

Will Yang, MA,

Visual Information Specialist

\section{MMWR Editorial Board}

Timothy F. Jones, MD, Chairman

William E. Halperin, MD, DrPH, MPH

Jewel Mullen, MD, MPH, MPA

Jeff Niederdeppe, $\mathrm{PhD}$

Celeste Philip, MD, MPH

Patricia Quinlisk, MD, MPH

Patrick L. Remington, MD, MPH
Carlos Roig, MS, MA

William Schaffner, MD

Nathaniel Smith, MD, MPH

Morgan Bobb Swanson, BS

Abbigail Tumpey, MPH 
TABLE 1. Prevalence of subjective cognitive decline* among unpaid adult caregivers ${ }^{\dagger}$ aged $\geq 45$ years, by state - Behavioral Risk Factor Surveillance System, 22 states, $\$$ 2015-2019

\begin{tabular}{lccr}
\hline State & $\begin{array}{c}\text { No. of } \\
\text { respondents who } \\
\text { are caregivers }\end{array}$ & $\begin{array}{c}\text { Estimated } \\
\text { (weighted) } \\
\text { no. of caregivers }\end{array}$ & $\begin{array}{c}\text { Weighted \% \% } \\
\text { with SCD } \\
(\mathbf{9 5 \%} \text { Cl) }\end{array}$ \\
\hline Overall & $\mathbf{2 1 , 2 3 8}$ & $\mathbf{1 2 , 6 9 3 , 0 0 0}$ & $\mathbf{1 2 . 6 ( 1 1 . 7 - 1 3 . 5 )}$ \\
Alabama & 1,257 & 465,000 & $14.1(11.6-16.7)$ \\
Florida & 683 & $1,864,000$ & $14.6(11.2-18.0)$ \\
Hawaii & 898 & 99,000 & $12.0(9.3-14.7)$ \\
Illinois & 665 & 975,000 & $10.1(7.5-12.6)$ \\
lowa & 692 & 191,000 & $10.6(7.9-13.2)$ \\
Louisiana & 736 & 415,000 & $17.3(13.8-20.8)$ \\
Maryland & 1,029 & 585,000 & $11.8(9.3-14.3)$ \\
Mississippi & 934 & 248,000 & $15.7(12.4-18.9)$ \\
Missouri & 913 & 464,000 & $15.5(11.6-19.3)$ \\
Montana & 734 & 78,000 & $13.5(9.8-17.1)$ \\
Nebraska & 1,510 & 181,000 & $11.3(8.9-13.8)$ \\
New Jersey & 396 & 720,000 & $9.8(5.3-14.3)$ \\
New York & 634 & $1,482,000$ & $11.1(7.6-14.5)$ \\
Oregon & 783 & 355,000 & $13.7(10.3-17.0)$ \\
South Carolina & 1,716 & 430,000 & $15.3(12.9-17.6)$ \\
Tennessee & 1,005 & 664,000 & $12.7(10.1-15.3)$ \\
Texas & 1,767 & $1,877,000$ & $11.8(9.3-14.2)$ \\
Utah & 798 & 234,000 & $14.9(11.8-17.8)$ \\
Virginia & 1,434 & 704,000 & $11.0(9.0-13.0)$ \\
West Virginia & 948 & 195,000 & $11.1(8.7-13.5)$ \\
Wisconsin & 765 & 413,000 & $14.3(10.8-17.8)$ \\
Wyoming & 941 & 54,000 & $11.9(9.0-14.9)$ \\
\hline Abbran & &
\end{tabular}

Abbreviation: $\mathrm{SCD}=$ subjective cognitive decline.

* SCD was defined as the self-reported experience of worsening confusion or memory loss in the past year.

${ }^{\dagger}$ Caregiving was defined as providing care to a family member or friend with a health condition or disability in the past 30 days.

$\S$ The following 22 U.S. states that included both caregiving and SCD modules in the same survey year during 2015-2019 are included (most recent year used): Alabama (2015), Florida (2015), Hawaii (2017), Illinois (2015), lowa (2015), Louisiana (2015), Maryland (2019), Mississippi (2015), Missouri (2016), Montana (2016), Nebraska (2015), New Jersey (2018), New York (2019), Oregon (2019), South Carolina (2015), Tennessee (2019), Texas (2019), Utah (2019), Virginia (2019), West Virginia (2015), Wisconsin (2015), and Wyoming (2015).

ๆ Estimates are weighted to each state's adult population.

between caregivers and noncaregivers by history of depression, number of days physical or mental health was not good in the past 30 days, or number of days health prevented regular activities in the past 30 days. Compared with noncaregivers, SCD prevalence among caregivers was higher among persons aged 45-64 years, men, non-Hispanic White persons, employed persons, persons who reported any chronic condition, and persons who reported good, very good, or excellent health.

The distribution of caregiver characteristics varied by SCD status (Table 3). Compared with caregivers without SCD, those with SCD were more likely to have at least one chronic condition, ${ }^{\dagger \dagger}$ a history of depression, report fair or poor health,

\footnotetext{
$\dagger_{\dagger}$ Any chronic condition was determined by an affirmative response to the question, "Has a doctor or other health professional ever told you that you had any of the following? For each, tell me Yes, No, or You're Not Sure: asthma (current); heart attack, angina, or coronary heart disease; a stroke; cancer other than skin cancer; chronic obstructive pulmonary disease, emphysema, or chronic bronchitis; some form of arthritis, rheumatoid arthritis, gout, lupus, or fibromyalgia; kidney disease, not including kidney stones, bladder infections, or incontinence; or diabetes, not including gestational, borderline, or prediabetes?"
}

report $\geq 14$ days of poor physical health in the past 30 days, report $\geq 14$ days of poor mental health in the past 30 days, and report $\geq 14$ days that health prevented regular activities in the past 30 days. Household status, duration or type of care provided, or the care recipient having a diagnosis of Alzheimer's disease, dementia, or other cognitive impairment disorder did not differ by SCD status.

\section{Discussion}

Among caregivers aged $\geq 45$ years in 22 participating states, approximately one in eight reported SCD, the self-reported experience of worsening or more frequent confusion or memory loss over the past year. SCD was more common in caregivers than in noncaregivers, particularly among those aged 45-64 years. SCD likely affects the quality and safety of care that caregivers can provide. Caregivers with SCD more frequently experienced negative physical and mental health than did caregivers without SCD. In addition, caregivers with SCD more frequently reported chronic conditions, being employed, being men, and were younger than noncaregivers with SCD, suggesting specific opportunities for interventions among caregivers with SCD. These findings are consistent with studies that indicate that, although there are benefits to caregiving, it can negatively affect a caregiver's physical and mental health (2-4). Adverse health outcomes have been found to be related to physical, emotional, and financial strains placed on caregivers, prioritization of care recipients' needs over caregivers' needs, and changes in behaviors that support caregivers' health such as delaying medical care or decreased physical activity (2-4).

As the U.S. population continues to age ( 6 ), the number of persons needing care is expected to increase. SCD among caregivers might make it more difficult to help care recipients manage medications, finances, or other aspects of their chronic conditions or health needs that require cognitive focus. Whether a caregiver with SCD can provide the level of support that is needed, and if so, for how long, are important considerations. Limitations in functional activities because of SCD might result in the need for assistance (5). Caregivers might need support themselves, both currently and in the future, especially given that this study found that more caregivers than noncaregivers experience SCD. SCD might be a symptom of early-stage dementia or a sign that more serious cognitive decline will occur in the future. SCD might also be a result of other health conditions that could be treatable, such as infections, medication interactions, or nutritional deficits, and potentially remain stable over time $(7,8)$. Caregivers are a crucial component of a caregiving team; however, these data suggest that caregivers, particularly those with SCD, might need support for their own health and well-being challenges. 
TABLE 2. Percentage of subjective cognitive decline* among unpaid caregivers ${ }^{\dagger}$ and noncaregivers aged $\geq 45$ years, by selected characteristics Behavioral Risk Factor Surveillance System, 22 states, $\$ 2015-2019$

\begin{tabular}{|c|c|c|c|c|c|}
\hline Characteristic & $\begin{array}{l}\text { Total unweighted } \\
\text { no. of } \\
\text { caregivers }\end{array}$ & $\begin{array}{c}\text { Caregivers with SCD, } \\
\text { weighted }^{* *} \\
\%(95 \% \mathrm{Cl})\end{array}$ & $\begin{array}{l}\text { Total unweighted } \\
\text { no. of } \\
\text { noncaregivers }\end{array}$ & $\begin{array}{l}\text { Noncaregivers with } \\
\text { SCD, weighted } \\
\%(95 \% \mathrm{Cl})\end{array}$ & $p$-value ${ }^{t \dagger}$ \\
\hline Overall & 21,238 & $12.6(11.7-13.5)$ & 72,366 & $10.2(9.7-10.7)$ & $<0.001$ \\
\hline \multicolumn{6}{|l|}{ Demographic characteristic } \\
\hline \multicolumn{6}{|l|}{ Age group, yrs } \\
\hline $45-64$ & 12,049 & $12.4(11.3-13.6)$ & 34,858 & $9.4(8.8-10.0)$ & $<0.001$ \\
\hline$\geq 65$ & 9,189 & $13.0(11.7-14.4)$ & 37,508 & $11.4(10.7-12.1)$ & 0.03 \\
\hline \multicolumn{6}{|l|}{ Sex } \\
\hline Men & 7,615 & $13.5(12.1-15.0)$ & 31,370 & $9.4(8.8-10.1)$ & $<0.001$ \\
\hline Women & 13,623 & $12.0(10.9-13.2)$ & 40,993 & $10.9(10.2-11.6)$ & 0.09 \\
\hline \multicolumn{6}{|l|}{ Race/Ethnicity } \\
\hline White, non-Hispanic & 16,689 & $12.9(11.9-13.9)$ & 56,555 & $9.7(9.2-10.2)$ & $<0.001$ \\
\hline Black, non-Hispanic & 2,226 & $12.0(9.5-14.5)$ & 7,184 & $12.7(11.1-14.3)$ & 0.6 \\
\hline $\begin{array}{l}\text { Asian/Pacific Islander, American Indian/Alaska Native, Other } \\
\text { race/Multiracial, non-Hispanic } \$ \S\end{array}$ & 1,312 & $14.3(8.9-19.8)$ & 4,476 & $9.9(7.9-11.9)$ & 0.1 \\
\hline Hispanic & 673 & $9.6(6.2-13.0)$ & 2,963 & $10.8(8.9-12.7)$ & 0.6 \\
\hline \multicolumn{6}{|l|}{ Education level } \\
\hline High school graduate or less & 7,041 & $15.0(13.3-16.6)$ & 27,920 & $7.9(7.4-8.3)$ & 0.06 \\
\hline Some college or more & 14,160 & $11.3(10.3-12.3)$ & 44,217 & $13.2(12.4-14.1)$ & $<0.001$ \\
\hline \multicolumn{6}{|l|}{ Employment status } \\
\hline Employed/Self-employed & 8,933 & $7.6(6.5-8.7)$ & 27,914 & $4.8(4.3-5.2)$ & $<0.001$ \\
\hline Unemployed & 829 & $21.0(14.6-27.4)$ & 1,994 & $14.8(11.6-18.0)$ & 0.09 \\
\hline Unable to work & 1,900 & $37.2(33.1-41.4)$ & 6,869 & $31.5(29.1-33.8)$ & 0.01 \\
\hline Other ${ }^{91}$ & 9,460 & $11.5(10.3-12.6)$ & 35,185 & $10.4(9.7-11.1)$ & 0.1 \\
\hline \multicolumn{6}{|l|}{ Health-related characteristic } \\
\hline \multicolumn{6}{|l|}{ Any chronic condition*** } \\
\hline Yes & 14,302 & $16.4(15.1-17.6)$ & 47,206 & $13.4(13.1-14.5)$ & $<0.001$ \\
\hline No & 6,777 & $5.7(4.6-6.7)$ & 24,620 & $4.3(3.8-4.8)$ & 0.02 \\
\hline \multicolumn{6}{|l|}{ History of depression } \\
\hline Yes & 4,915 & $28.3(25.8-30.8)$ & 12,582 & $27.2(25.9-29.4)$ & 0.7 \\
\hline No & 16,239 & $8.0(7.2-8.9)$ & 59,462 & $6.8(6.4-7.2)$ & 0.01 \\
\hline \multicolumn{6}{|l|}{ General health status } \\
\hline Good, very good, or excellent & 16,454 & $8.1(7.4-9.0)$ & 55,300 & $6.1(5.7-6.5)$ & $<0.001$ \\
\hline Fair or poor & 4,734 & $26.8(24.3-29.4)$ & 16,855 & $23.3(21.9-24.8)$ & 0.02 \\
\hline \multicolumn{6}{|l|}{ No. of days physical health was not good in past 30 days } \\
\hline None & 12,106 & $6.5(5.7-7.4)$ & 43,638 & $5.1(4.7-5.5)$ & 0.003 \\
\hline $1-13$ & 5,196 & $15.2(13.2-17.1)$ & 14,985 & $13.2(12.0-14.5)$ & 0.09 \\
\hline$\geq 14$ & 3,550 & $28.2(25.2-31.1)$ & 11,878 & $24.7(23.0-26.2)$ & 0.04 \\
\hline \multicolumn{6}{|l|}{ No. of days mental health was not good in past 30 days } \\
\hline None & 13,363 & $6.2(5.5-6.9)$ & 52,915 & $5.5(5.1-5.9)$ & 0.1 \\
\hline $1-13$ & 4,692 & $16.1(14.0-18.1)$ & 11,691 & $14.7(13.4-16.0)$ & 0.3 \\
\hline$\geq 14$ & 2,853 & $34.4(30.7-38.0)$ & 6,424 & $36.5(33.9-39.2)$ & 0.3 \\
\hline \multicolumn{6}{|l|}{ No. of days health prevented regular activities in past 30 days } \\
\hline None & 6,469 & $11.4(9.8-13.0)$ & 18,832 & $9.5(8.7-10.3)$ & 0.03 \\
\hline $1-13$ & 3,159 & $19.0(16.4-21.7)$ & 8,211 & $16.5(14.7-18.2)$ & 0.1 \\
\hline$\geq 14$ & 2,340 & $33.9(30.1-37.8)$ & 7,615 & $33.8(31.4-36.2)$ & 0.9 \\
\hline
\end{tabular}

Abbreviation: $\mathrm{SCD}=$ subjective cognitive decline.

* SCD was defined as the self-reported experience of worsening confusion or memory loss in the past year.

+ Caregiving was defined as providing care to a family member or friend with a health condition or disability in the past 30 days.

$\S$ The following 22 U.S. states that included both caregiving and SCD modules in the same survey year during 2015-2019 are included (most recent year used): Alabama (2015), Florida (2015), Hawaii (2017), Illinois (2015), lowa (2015), Louisiana (2015), Maryland (2019), Mississippi (2015), Missouri (2016), Montana (2016), Nebraska (2015), New Jersey (2018), New York (2019), Oregon (2019), South Carolina (2015), Tennessee (2019), Texas (2019), Utah (2019), Virginia (2019), West Virginia (2015), Wisconsin (2015), and Wyoming (2015).

" Categories might not sum to the sample total because of missing responses.

** Estimates are weighted to each state's adult population.

${ }^{\dagger+} \mathrm{T}$-tests were used to determine statistically significant differences between caregivers and noncaregivers with SCD for each level of selected characteristics at $\mathrm{p}<0.05$.

$\S$ Asian/Pacific Islander, American Indian/Alaska Native, and Other or multiracial non-Hispanic persons were combined into one group because of small sample sizes.

११ Homemaker, student, or retired.

*** Any chronic condition was determined by an affirmative response to the question, "Has a doctor or other health professional ever told you that you had any of the following? For each, tell me Yes, No, or You're Not Sure: asthma (current); heart attack, angina, or coronary heart disease; a stroke; cancer other than skin cancer; chronic obstructive pulmonary disease, emphysema, or chronic bronchitis; some form of arthritis, rheumatoid arthritis, gout, lupus, or fibromyalgia; kidney disease, not including kidney stones, bladder infections, or incontinence; or diabetes, not including gestational, borderline, or prediabetes?" 
TABLE 3. Distribution of selected characteristics among unpaid caregivers* aged $\geq 45$ years by subjective cognitive decline status ${ }^{\dagger}-$ Behavioral $^{*}$ Risk Factor Surveillance System, 22 states, $\$$ 2015-2019

\begin{tabular}{|c|c|c|c|}
\hline \multirow[b]{2}{*}{ Characteristic } & \multicolumn{2}{|c|}{ Caregivers, weighted" \% $(95 \% \mathrm{Cl})$} & \multirow[b]{2}{*}{$p$-value $* *$} \\
\hline & With SCD $(n=2,670)$ & Without SCD $(n=18,568)$ & \\
\hline $\begin{array}{l}\text { Household status } \\
\text { Lives alone } \\
\text { Does not live alone }\end{array}$ & $\begin{array}{l}20.0(17.0-23.0) \\
80.0(77.0-83.0)\end{array}$ & $\begin{array}{r}17.6(16.5-18.90 \\
82.4(81.4-83.5)\end{array}$ & 0.1 \\
\hline Health-related characteristic & & & \\
\hline $\begin{array}{l}\text { Any chronic condition }{ }^{\dagger+} \\
\text { Yes } \\
\text { No }\end{array}$ & $\begin{array}{l}84.7(82.0-87.3) \\
15.3(12.7-18.0)\end{array}$ & $\begin{array}{l}62.9(61.4-64.4) \\
37.1(35.6-38.6)\end{array}$ & $<0.001$ \\
\hline $\begin{array}{l}\text { History of depression } \\
\text { Yes } \\
\text { No }\end{array}$ & $\begin{array}{l}50.3(46.6-54.0) \\
49.7(46.0-53.4)\end{array}$ & $\begin{array}{l}18.3(17.1-19.4) \\
81.7(80.6-82.9)\end{array}$ & $<0.001$ \\
\hline $\begin{array}{l}\text { General health status } \\
\text { Good, very good, or excellent } \\
\text { Fair or poor }\end{array}$ & $\begin{array}{l}49.3(45.6-53.0) \\
50.7(47.0-54.4)\end{array}$ & $\begin{array}{l}80.0(78.7-81.2) \\
20.0(18.8-21.3)\end{array}$ & $<0.001$ \\
\hline $\begin{array}{l}\text { No. of days physical health was not good in past } 30 \mathrm{c} \\
\text { None } \\
1-13 \\
\geq 14\end{array}$ & $\begin{array}{l}29.2(25.8-32.5) \\
31.7(28.1-35.2) \\
39.2(35.5-42.8)\end{array}$ & $\begin{array}{l}60.2(58.6-61.7) \\
25.5(24.1-26.8) \\
14.4(13.3-15.5)\end{array}$ & $<0.001$ \\
\hline $\begin{array}{l}\text { No. of days mental health was not good in past } 30 \mathrm{~d} d \\
\text { None } \\
1-13 \\
\geq 14\end{array}$ & $\begin{array}{l}30.0(26.9-33.2) \\
30.7(27.2-34.1) \\
39.3(35.5-43.1)\end{array}$ & $\begin{array}{r}66.0(64.5-67.5) \\
23.2(21.8-24.5) \\
10.8(9.9-11.8)\end{array}$ & $<0.001$ \\
\hline $\begin{array}{l}\text { No. of days health prevented regular activities in pas } \\
\text { None } \\
1-13 \\
\geq 14\end{array}$ & $\begin{array}{l}33.8(29.8-37.7) \\
29.0(25.3-32.6) \\
37.3(33.4-41.2)\end{array}$ & $\begin{array}{l}57.3(55.2-59.3) \\
26.9(25.0-28.8) \\
15.8(14.2-17.4)\end{array}$ & $<0.001$ \\
\hline $\begin{array}{l}\text { Main health condition of care recipient } \\
\text { Alzheimer's disease/Cognitive impairment/Dementia } \\
\text { All other health conditions }\end{array}$ & $\begin{array}{l}12.3(10.0-14.6) \\
87.7(85.4-90.0)\end{array}$ & $\begin{array}{l}12.8(11.8-13.8) \\
87.2(86.2-88.2)\end{array}$ & 0.7 \\
\hline $\begin{array}{l}\text { Length of care provided, yrs } \\
<5 \\
\geq 5\end{array}$ & $\begin{array}{l}66.3(62.8-69.7) \\
33.7(30.3-37.2)\end{array}$ & $\begin{array}{l}67.6(66.0-69.2) \\
32.4(30.8-87.0)\end{array}$ & 0.5 \\
\hline $\begin{array}{l}\text { No. of weekly hours of care provided } \\
<20 \\
\geq 20\end{array}$ & $\begin{array}{l}65.0(61.3-68.8) \\
35.0(31.2-38.7)\end{array}$ & $\begin{array}{l}68.7(67.2-70.2) \\
31.3(29.8-32.8)\end{array}$ & 0.08 \\
\hline $\begin{array}{l}\text { Type of assistance provided } \\
\text { Personal care only } \$ \S \\
\text { Household tasks only } \\
\text { Personal care and household tasks } \\
\text { Neither personal care nor household tasks }\end{array}$ & $\begin{array}{r}7.9(5.7-10.1) \\
37.6(33.9-41.3) \\
54.5(50.6-58.4) \\
19.0(15.8-22.3)\end{array}$ & $\begin{array}{r}6.9(6.0-7.8) \\
38.5(36.9-40.2) \\
54.6(52.9-56.3) \\
18.0(16.8-19.2)\end{array}$ & 0.8 \\
\hline
\end{tabular}

Abbreviation: SCD = subjective cognitive decline.

* Caregiving was defined as providing care to a family member or friend with a health condition or disability in the past 30 days.

† SCD was defined as the self-reported experience of worsening confusion or memory loss in the past year.

$\S$ The following 22 U.S. states that included both caregiving and SCD modules in the same survey year during 2015-2019 are included (most recent year used): Alabama (2015), Florida (2015), Hawaii (2017), Illinois (2015), lowa (2015), Louisiana (2015), Maryland (2019), Mississippi (2015), Missouri (2016), Montana (2016), Nebraska (2015), New Jersey (2018), New York (2019), Oregon (2019), South Carolina (2015), Tennessee (2019), Texas (2019), Utah (2019), Virginia (2019), West Virginia (2015), Wisconsin (2015), and Wyoming (2015).

I Estimates are weighted to each state's adult population.

** P-values from chi-square analyses measure the association between proportions, with modified Rao-Scott chi-square tests.

${ }^{\dagger+}$ Any chronic condition was determined by an affirmative response to the question, "Has a doctor or other health professional ever told you that you had any of the following? For each, tell me Yes, No, or You're Not Sure: asthma (current); heart attack, angina, or coronary heart disease; a stroke; cancer other than skin cancer; chronic obstructive pulmonary disease, emphysema, or chronic bronchitis; some form of arthritis, rheumatoid arthritis, gout, lupus, or fibromyalgia; kidney disease, not including kidney stones, bladder infections, or incontinence; or diabetes, not including gestational, borderline, or prediabetes?"

$\S \S$ Personal care tasks were defined as administering medications, feeding, dressing, or bathing.

११ Household tasks were defined as cleaning, managing money, or preparing meals. 


\section{Summary}

What is already known about this topic?

Caregiving can negatively affect caregivers' physical and mental health. Little is known about caregivers' cognitive functioning.

What is added by this report?

Among unpaid adult caregivers aged $\geq 45$ years, approximately one in eight reported subjective cognitive decline (SCD) (the self-reported experience of worsening confusion or memory loss over the past year). SCD was higher among caregivers (12.6\%) than among noncaregivers (10.2\%). Caregivers with SCD were more likely than those without SCD to report chronic health conditions, a history of depression, and frequent activity limitations.

What are the implications for public health practice?

SCD among caregivers could affect the quality of care provided to care recipients. Health care professionals can support their patients and their patients' caregivers by recognizing SCDassociated challenges in providing care and providing compensatory strategies to promote the health and well-being of caregivers and their care recipients.

The findings in this report are subject to at least four limitations. First, causality between caregiving and SCD cannot be inferred from a cross-sectional study. Second, self-reported data might be subject to several biases, including recall and social desirability biases, which might result in under- or overreporting of SCD. Third, these data cannot be validated with medical examination records, but the perception of decline (versus objectively measured decline) is associated with development of Alzheimer's disease or other dementias $(9,10)$. Finally, with data from 22 states, the findings of this report cannot be extrapolated to the rest of the country. A major strength of this study is the large sample size of caregivers.

Considering the growth of the older adult population, the increased prevalence of dementia, and an increasing need for caregiving, understanding the cognitive health and needs of caregivers to better support them and their care recipients is critical. Unpaid caregivers are an essential facet of a caregiving team; however, these data suggest that caregivers might also need support for their own cognitive and physical health and well-being. Health care professionals can support their patients and their patients' caregivers by recognizing SCD and its associated challenges in providing care and providing compensatory strategies to promote the health and well-being of both caregivers and their care recipients. ${ }^{\$ \$}$ Public health professionals can continue working to support caregivers and care recipients throughout the caregiving process by strengthening public health infrastructure utilizing the public health strategist approach 99 and resources such as evidence-based interventions and training materials from CDC's Building Our Largest Dementia Infrastructure Public Health Center of Excellence on Dementia Caregiving. ${ }^{* * *}$

*** https://bolddementiacaregiving.org/

Corresponding author: Eva M. Jeffers, EJeffers@cdc.gov, 404-498-4309.

${ }^{1}$ Division of Population Health, National Center for Chronic Disease Prevention and Health Promotion, CDC; ${ }^{2}$ Oak Ridge Institute for Science and Education, Oak Ridge, Tennessee; ${ }^{3}$ Department of Health and Exercise Science, Appalachian State University, Boone, North Carolina; ${ }^{4}$ Department of Public Health and Center for Long-Term Care, New York Medical College, Valhalla, New York; ${ }^{5}$ CyberData Technologies, Inc., Herndon, Virginia.

All authors have completed and submitted the International Committee of Medical Journal Editors form for disclosure of potential conflicts of interest. No potential conflicts of interest were disclosed.

\section{References}

1. Edwards VJ, Bouldin ED, Taylor CA, Olivari BS, McGuire LC. Characteristics and health status of informal unpaid caregivers-44 states, District of Columbia, and Puerto Rico, 2015-2017. MMWR Morb Mortal Wkly Rep 2020;69:183-8. PMID:32078592 https://doi. org/10.15585/mmwr.mm6907a2

2. Committee on Family Caregiving for Older Adults; Board on Health Care Services; National Academies of Sciences, Engineering, and Medicine. Families caring for an aging America. Schulz R, Eden J, eds. Washington, DC: The National Academies Press (US); 2016. https:// www.ncbi.nlm.nih.gov/books/NBK396401/

3. Schulz R, Sherwood PR. Physical and mental health effects of family caregiving. Am J Nurs 2008;108(Suppl):23-7, quiz 27. PMID:18797217 https://doi.org/10.1097/01.NAJ.0000336406.45248.4c

4. Miyawaki CE, Bouldin ED, Taylor CA, McGuire LC. Baby boomers as caregivers: results from the Behavioral Risk Factor Surveillance System in 44 states, the District of Columbia, and Puerto Rico, 2015-2017. Prev Chronic Dis 2020;17:E80. PMID:32790608 https://doi. org/10.5888/pcd17.200010

5. Taylor CA, Bouldin ED, McGuire LC. Subjective cognitive decline among adults aged $\geq 45$ years-United States, 2015-2016. MMWR Morb Mortal Wkly Rep 2018;67:753-7. PMID:30001562 https://doi. org/10.15585/mmwr.mm6727a1

6. Ortman JM, Velkoff VA, Hogan H. An aging nation: the older population in the United States. Washington, DC: US Department of Commerce, US Census Bureau; 2014. https://www.census.gov/ $\mathrm{prod} / 2014 \mathrm{pubs} / \mathrm{p} 25-1140 . \mathrm{pdf}$

7. Alzheimer's Association. 2018 Alzheimer's disease facts and figures. Alzheimers Dement 2018;14:367-429. https://doi.org/10.1016/j. jalz.2018.02.001

8. Kaup AR, Nettiksimmons J, LeBlanc ES, Yaffe K. Memory complaints and risk of cognitive impairment after nearly 2 decades among older women. Neurology 2015;85:1852-8. PMID:26511452 https://doi. org/10.1212/WNL.0000000000002153

9. Valech N, Mollica MA, Olives J, et al. Informants' perception of subjective cognitive decline helps to discriminate preclinical Alzheimer's disease from normal aging. J Alzheimers Dis 2015;48(Suppl 1):S87-98. PMID:26445275 https://doi.org/10.3233/JAD-150117

10. Perrotin A, de Flores R, Lamberton F, et al. Hippocampal subfield volumetry and $3 \mathrm{D}$ surface mapping in subjective cognitive decline. J Alzheimers Dis 2015;48(Suppl 1):S141-50. PMID:26402076 https:// doi.org/10.3233/JAD-150087

\footnotetext{
$\overline{\$ \$}$ https:/www.cdc.gov/aging/data/subjective-cognitive-decline-brief.html

99 https://www.cdc.gov/aging/caregiving/caregiver-brief.html
} 